\title{
Neoclassical resonant transport of a mirror cell
}

\author{
T. Ito and I. Katanuma ${ }^{\text {a) }}$ \\ Plasma Research Center, University of Tsukuba, Tsukuba, Ibaraki 305-8577, Japan
}

(Received 18 May 2005; accepted 7 July 2005; published online 16 August 2005)

The neoclassical resonant plateau transport in a mirror cell is studied theoretically. The analytical expression for a non-square-well magnetic field is obtained. The analytical result is applied to the GAMMA10 tandem mirror [T. Cho, M. Yoshida, J. Kohagura et al., Phys. Rev. Lett. 94, 085002-1 (2005)], which consists of several mirror cells in it, and the confinement time due to the neoclassical resonant plateau transport is determined in each mirror cell. It is found that the neoclassical resonant transport of ions trapped in the nonaxisymmetric anchor mirror cell and transition mirror cells is significantly smaller than those trapped in the central cell. (C) 2005 American Institute of Physics.

[DOI: $10.1063 / 1.2009547]$

\section{INTRODUCTION}

In a conventional tandem mirror system ${ }^{1,2}$ the radial transport is caused by a diffusive process of the second adiabatic invariant $J,{ }^{3,4}$ where the process related to the resonant interactions between the ion azimuthal drift frequency and the axial bounce frequency leads to diffusion in $J$, resulting in a radial transport in a tandem mirror containing nonaxisymmetric quadrupole end-mirror cells.

In axisymmetric mirror fields, where the azimuthal drift velocities are constant and the drift surfaces are circular, there are no such resonances, so that the second adiabatic invariant $J$ conserves in the collisionless or collective processes, and radial transport stemming from nonadiabaticity does not occur. In nonaxisymmetric mirror cells such as a tandem mirror with quadrupole minimum- $B$ end-mirror cells, the quadrupole magnets closely employed to the central cell distort the axisymmetry of that cell. In such nonaxisymmetric mirror cells azimuthal drift velocities are not constant but vary with the azimuth, so that bounce-drift harmonic resonances can occur. While collisions can modify the rate of the radial transport stemming from the diffusion processes, it is recognized that their origin is in the basic properties of the single-particle orbits characteristic of the confining fields. ${ }^{5,6}$

The main reason that a tandem mirror contains the nonaxisymmetric quadrupole mirror cells is for the magnetohydrodynamic (MHD) stability. ${ }^{7,8}$ However, the quadrupole mirror cells located at both ends of a tandem mirror can distort the magnetic-flux tube in the central cell in the case of the finite plasma pressure, ${ }^{9-11}$ which enhances the radial transport even in the axisymmetric central cell of a tandem mirror. The recent study of a tandem mirror design, therefore, is a fully axisymmetric tandem mirror, where a divertor magnetic configuration is installed around the central cell midplain for the stability of MHD. The theoretical study of MHD stability for the magnetic divertor of a tandem mirror was first carried out by Lane et al. within the long thin approximation ${ }^{12}$ and Pastukhov et al. improved the theory to that without the long thin approximation. ${ }^{13}$ This design of a magnetic divertor is a latest problem in improving the stabil-

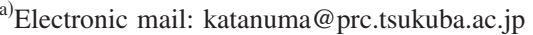

ity and radial confinement of the GAMMA10 tandem mirror ${ }^{14}$ with the developments in the theoretical study of the divertor physics. ${ }^{15,16}$

The analysis of the radial transport effects in a conventional tandem mirror containing nonaxisymmetric mirror cells is described with a consideration of the motion of the guiding center of particles, bouncing axially and drifting azimuthally in the mirror cells. In the limit of infinitesimal particle gyroradius $\rho_{i}$, the guiding center of the particle would retain on precise drift surfaces, but this is not quite the case when the gyroradius is finite. In this case the guiding-center motion is averaged over the gyrating motion of the local magnetic field, which in a nonaxisymmetric field has gradients in three directions, axial, radial, and azimuthal, respectively. Accordingly a particle starting at the midplane in a mirror cell being reflected, and returning to the midplane will have its guiding center displaced both azimuthally drift and radially inwards or outwards from its starting position. This displacement of the radial direction, defined as $\Delta r$, is a function of the magnetic moment $\mu$ and energy $\epsilon$ of the particle and also of the initial radius $r$ and azimuthal angle $\theta$ of its guiding center. For example Bernstein ${ }^{17}$ and $\mathrm{Cohen}^{18}$ described the drift equations for a static magnetic field $\boldsymbol{B}$ as

$$
\Delta r(\epsilon, \mu, r, \theta)=\frac{c}{e B R} \frac{\partial J}{\partial \theta},
$$

where $J$ is evaluated over the trajectory of the guiding center between the midplane and the turning point $z_{t}$ in a mirror cell, and described as

$$
J=\int_{0}^{z_{t}} \mathrm{~d} z[2 m(\epsilon-\mu B-e \phi)]^{1 / 2} .
$$

In a tandem mirror central cell, contributions to $\partial J / \partial \theta$ in Eq. (1) will arise only from the nonaxisymmetric transition regions at the ends because the central mirror cell is axisymmetric. If we assume the $\beta$ (plasma pressure divided by magnetic pressure) is small in these transition regions, then $J$ can be evaluated from the vacuum field configuration by expanding the integrand in Eq. (2) in powers of $r^{2}$, which is the radius of a vacuum field line. To leading order $r$ is written as $^{18,19}$ 


$$
\Delta r=\frac{\rho_{i} r}{L_{\mathrm{tr}}} K(\epsilon, \mu) \sin 2 \theta,
$$

where $L_{\mathrm{tr}}$ is the length of the transition region and $\rho_{i}$ is the ion Larmor radius; $K(\epsilon, \mu)$ is a complicated integral and requires numerical evaluation for particle-orbit tracing in a magnetic field.

One effect of the quadrupole fields is found from Eq. (3). The $\sin 2 \theta$ dependence reflects the fact that along lines having zero geodesic curvature (those planes where $\sin 2 \theta=0$ ), the radial displacement does not occur. The second effect of the quadrupole fields arises when the usual situation (fans displaced azimuthally $\pi / 2$ from each other, characteristic of a quadrupole field) is encountered. Here displacements of the radial direction on subsequent reflections cancel [through the order $\left.\left(r / L_{\mathrm{tr}}\right)^{2}\right]$. This cancelation results from the fact that reflections at each end differ in $\theta$ by $\pi / 2$ from the previous reflection, as far as orientation in the fans is concerned. Accordingly, for the $(n+1)$ th reflection, we have, relative to the $n$th reflection, $\sin 2 \theta_{n+1}=\sin 2\left(\theta_{n}+\pi / 2\right)=-\sin 2 \theta_{n}$, i.e., the $(n+1)$ th displacement cancels the $n$th displacement, and so on. In this cancelation, on average, there could be no radial transport apart from the influence of collisions or microscopic drifts.

Reality is introduced into the situation by considering the modifications caused by two effects with which, following Ryutov and Stupakov, ${ }^{19,20}$ one identifies two extreme regimes: regime 1 where $\Delta \theta \ll 1$, and regime 2 where $\Delta \theta \gtrsim 1$. Here $\Delta \theta$ is the amount of a particle azimuthal drift per one axial bounce motion. In regime 1 radial transport analogous to neoclassical transport in tokamaks occurs: Collisioninduced change in $\mu$ and $\epsilon$ moves the particles onto adjacent drift surfaces, leading to radial transport with an equivalent step size of the order of the banana width of the drift surfaces per effective collisions. As is the case in the tokamak, this neoclassical radial transport can be correspondingly larger than the classical rate of cross-field transport.

Regime 2 , where $\Delta \theta \gtrsim 1$, corresponds to a regime where the so-called resonant transport can occur. As noted previously, when the azimuthal drift per bounce is large enough to change the sign of the $\sin 2 \theta$ term at each bounce, successive radial displacements will add continuously, which means that "resonance" occurs. Resultant radial transport can then be enhanced substantially over the neoclassical rate. Regime 2 can arise when the azimuthal drifts associated with $\boldsymbol{E} \times \boldsymbol{B}$ drifts in radial ambipolar electric fields lead to large $\Delta \theta$ values.

\section{NEOCLASSICAL RESONANT TRANSPORT IN THE GAMMA10 TANDEM MIRROR}

In this section we extend the neoclassical resonant transport theory ${ }^{18-23}$ to calculate the ion radial flux in a nonaxisymmetric mirror. The diffusion coefficients are necessary to evaluate the particle loss attributed to the neoclassical resonant transport and some quantities of the particle motion are required in order to determine those coefficients. First we describe the basic equations to evaluate these quantities and the magnetic field of GAMMA10. ${ }^{24}$ We adopt the paraxial approximation to the magnetic field with the flux coordinates $(\psi, \theta, z)$, where the equations of motion are written as (for example, see Ref. 21)

$$
\begin{aligned}
& \frac{d \psi}{d t}=-\frac{c}{q} \kappa_{\theta}(2 \epsilon-\mu B-2 q \phi), \\
& \frac{d \theta}{d t}=c \frac{\partial \phi}{\partial \psi}+\frac{c}{q} \kappa_{\psi}(2 \epsilon-\mu B-2 q \phi), \\
& \frac{d z}{d t}=v_{\|}, \quad v_{\|}= \pm\left[\frac{2}{m_{i}}(\epsilon-\mu B-q \phi)\right]^{1 / 2} .
\end{aligned}
$$

Here the magnetic field $\boldsymbol{B}$ is represented by the coordinate variables as $\boldsymbol{B}=\nabla \psi \times \nabla \theta, z$ is the coordinate along the mirror axis defined as $z=0$ at the central cell midplane, $c$ is the light speed, $2 \pi \psi$ is the magnetic flux, i.e., $\psi=\frac{1}{2} r^{2} B_{0}$, where $B_{0}$ is the magnetic field at $z=0, \epsilon$ is the particle energy, $\mu$ is the magnetic moment, $q$ is the charge, $m_{i}$ is the ion mass, and $r$ is the radial distance in terms of the value at the central mirror cell midplane. The electrostatic potential $\phi$ is assumed to be a function of $\psi$ but independent of $\theta$ and $z$. The curvature of magnetic-field line $\boldsymbol{\kappa}$ is represented by $\boldsymbol{\kappa}$ $=\kappa_{\psi} \nabla \psi+\kappa_{\theta} \nabla \theta$, where $\kappa_{\psi}$ and $\kappa_{\theta}$ are the normal and geodesic curvatures with the following relations;

$$
\begin{aligned}
& \kappa_{\psi}=\frac{1}{2} \hat{\kappa}_{\psi}-\frac{1}{2} \hat{\kappa}_{\theta} \cos 2 \theta, \\
& \kappa_{\theta}=\hat{\kappa}_{\theta} \psi \sin 2 \theta, \\
& \hat{\kappa}_{\psi}=\frac{\sigma^{\prime \prime} \sigma+\tau^{\prime \prime} \tau}{B_{0}}, \quad \hat{\kappa}_{\theta}=-\frac{\sigma^{\prime \prime} \sigma-\tau^{\prime \prime} \tau}{B_{0}}, \\
& x=\sigma(z) x_{0}, \quad y=\tau(z) y_{0},
\end{aligned}
$$

where $(x, y)$ are the coordinates of the magnetic-field line starting at $\left(x_{0}, y_{0}\right)$ at the midplane in the central mirror cell; the prime denotes derivatives with respect to $z$.

Figure 1 shows the GAMMA10 magnetic field profile along the $z$ axis, where GAMMA10 consists of several mirror cells. The midplane of the minimum- $B$ anchor cell is located at $z= \pm 520 \mathrm{~cm}$. There are mirror cells called transition mirror cells next to the anchor mirror cell. The midplane of each transition mirror cell is located at $z= \pm 365 \mathrm{~cm}$ and $z= \pm 673 \mathrm{~cm}$. These transition mirror cells are labeled as "Transition Mirror Cell No. 1" and "Transition Mirror Cell No. 2." The normal curvature $\kappa_{\psi}$ and geodesic curvature $\kappa_{\theta}$ are designed to be symmetric and antisymmetric, respectively, around $z= \pm 520 \mathrm{~cm}$ to suppress the neoclassical transport shown in Fig. 1(b). The variables $\sigma$ and $\tau$ characterizing the magnetic-field line of GAMMA10 are defined in Eq. (5) and are shown in Fig. 1(c). A circular magnetic-flux tube starting at the midplane in the central cell $(z=0 \mathrm{~cm})$ is transformed into an elliptical shape in transition mirror cell No. 1 and first recircularized at the midplane in the anchor cell 
(a)

\section{GAMMA10 side view}
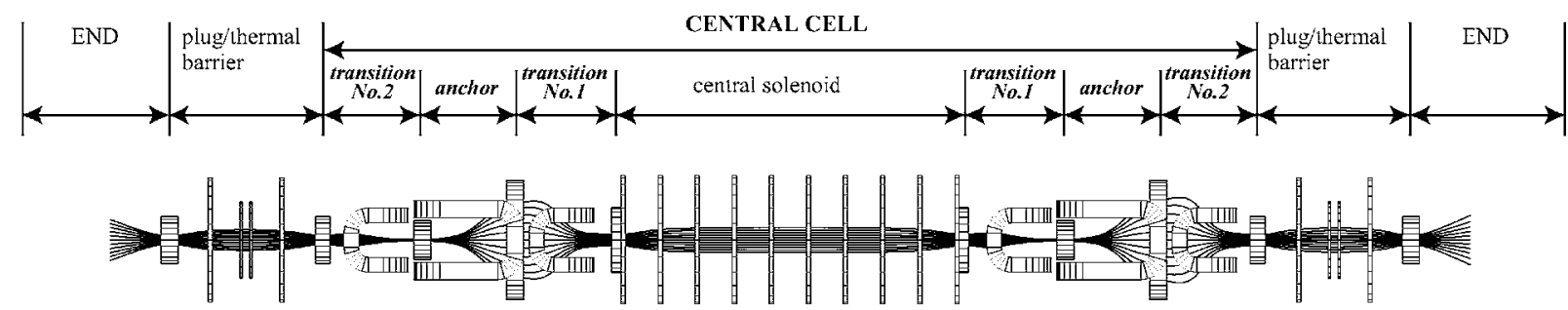

(b)

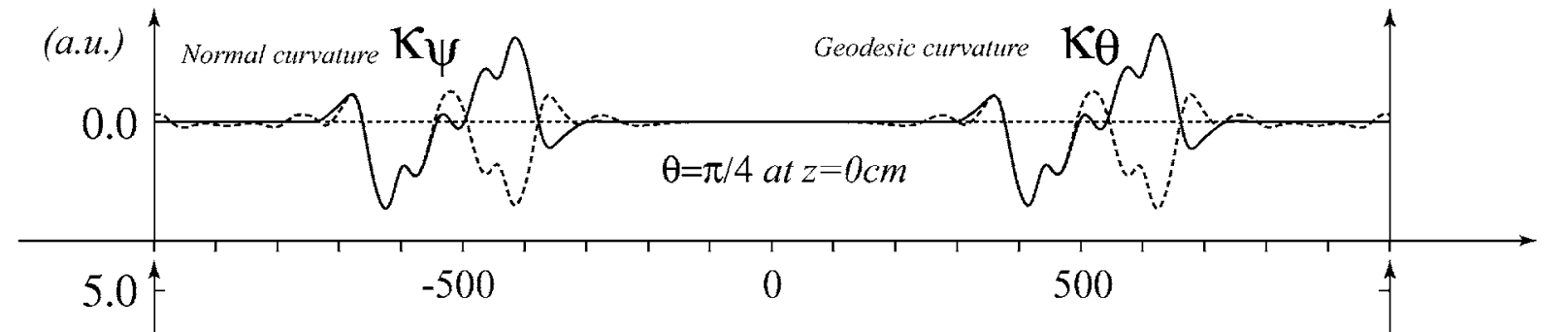

(d)
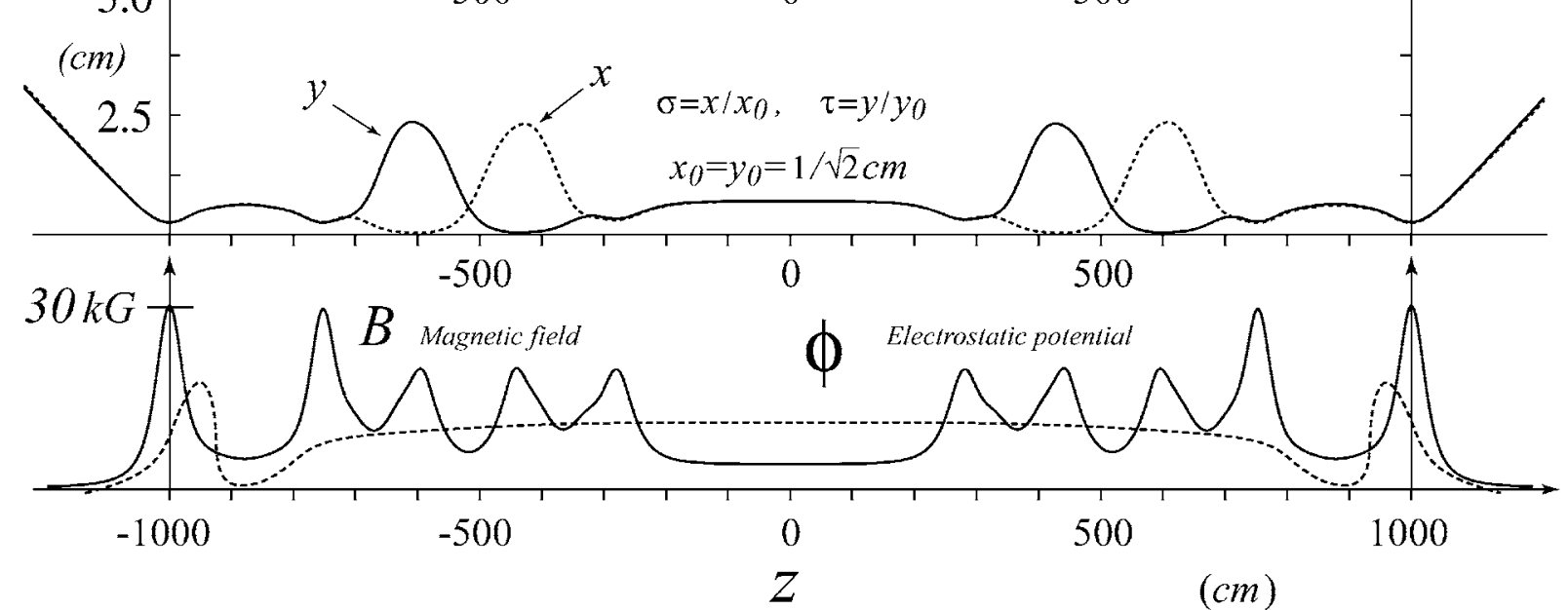

FIG. 1. Schematic diagram of the GAMMA10 tandem mirror. (a) Plots the GAMMA10 coils with magnetic-field lines. The labels of each mirror cell are also shown. (b) is the axial profiles of normal curvature $\kappa_{\psi}$ (dashed line) and geodesic curvature $\kappa_{\theta}$ (solid line), where these curvatures are those of the magnetic-field line passing through at $\theta=\pi / 4$ at $z=0$. (c) is a magnetic-field line which passes through at $x=y=1 / \sqrt{2} \mathrm{~cm}$ at the central cell midplane. (d) is the axial profiles of the magnitude of the magnetic field (solid line) and anticipated electrostatic potential (dashed line).

$(z=520 \mathrm{~cm})$, transformed next into an elliptical shape in transition mirror cell No. 2 , and then recircularized again at the midplane in the plug/barrier end cell $(z=880 \mathrm{~cm})$. The axial profiles of the magnetic field and the anticipated electrostatic potential $\phi$ are shown in Fig. 1(d), where the axial profile of $\phi$ is assumed to be constant, except for the plug/ thermal barrier mirror cells ${ }^{24-30}$ located at both ends of GAMMA10. The shape of the magnetic-flux tube and the names of the mirror cells in the nonaxisymmetric region are shown in Fig. 2.

\section{NEOCLASSICAL RESONANT PLATEAU TRANSPORT IN A MIRROR}

The conventional method of neoclassical resonant transport assumes that the axial length of a nonaxisymmetric magnetic field is much shorter than that of an axisymmetric central cell, so that the magnetic mirror field is approximated by a square well. However, it is difficult to apply this as- sumption to the nonaxisymmetric magnetic-field-like anchor mirror cells or transition mirror cells in GAMMA10. So the conventional neoclassical resonant plateau transport theory is extended to be applicable to the nonaxisymmetric magnetic field in this section.

We assume that $\Delta \psi$ is defined as the displacement of radial coordinate $\psi$ during an ion one-bounce axial motion in a mirror cell (from $z=z_{\text {start }}$ to $z=$ turning point 1 , back to $z_{\text {start }}$, to $z=$ turning point 2 , and back to $z_{\text {start }}$ ), and $\Delta \theta$ is defined as the displacement of azimuthal coordinate $\theta$ per ion onebounce axial motion. Anchor mirror cells and transition mirror cells are designed to be the nonaxisymmetric structure around the midplane in each mirror cell; the ions confined in those mirror cells are the cause of the large radial drift. Especially the ion that drifts at the amount of $\Delta \theta=k \pi, k=1,3$, $5, \ldots$, in the azimuthal direction per one-bounce axial motion comes under the strong influence of the nonaxisymmetric magnetic field and drifts radially. We call the ion satisfying this condition of $\Delta \theta=k \pi$ a "resonant ion."

Integrating Eq. (4), $\Delta \psi$, and $\Delta \theta$ in the zeroth-order orbit, 


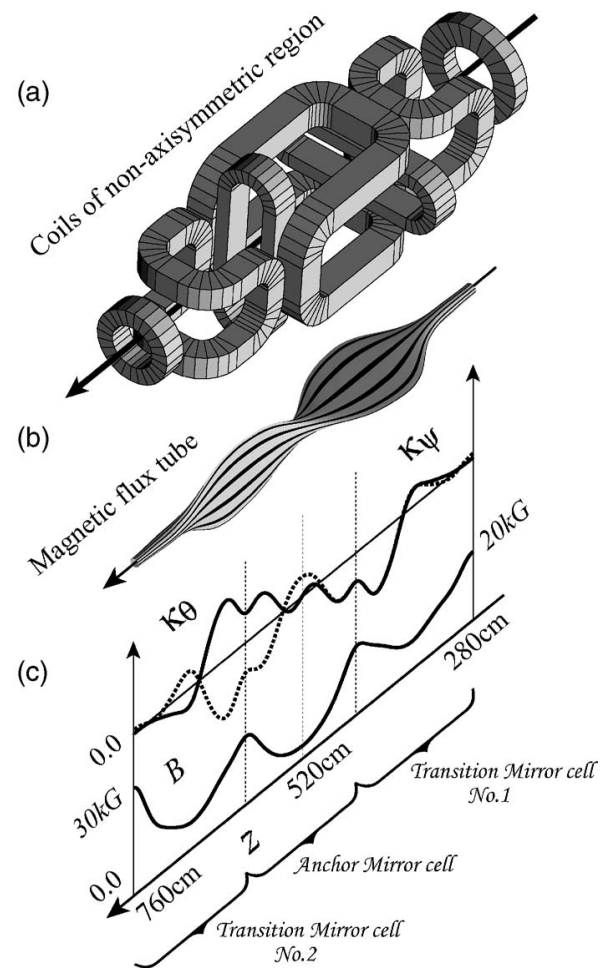

FIG. 2. Nonaxisymmetric region of the GAMMA10 tandem mirror. (a) is a birds-eye view of the coils in the nonaxisymmetric region. (b) is a magneticflux tube which is circular at the central cell midplane $z=0 \mathrm{~cm}$. The black stripe is along the magnetic-field lines. (c) Plots the axial profile magneticfield line curvatures and magnitude of the magnetic field. The label "Transition Mirror Cell No.1" is located in the region of $280 \mathrm{~cm}<|z|<445 \mathrm{~cm}$, "Anchor Mirror Cell" in the region of $445 \mathrm{~cm}<z<595 \mathrm{~cm}$, and "Transition Mirror Cell No. 2" in the region of $595 \mathrm{~cm}<z<755 \mathrm{~cm}$

$$
\begin{aligned}
\Delta \psi & =-\frac{c}{q} \oint_{\text {orbit }} \frac{d z}{v_{\|}} \hat{\kappa}_{\theta} \psi(2 \epsilon-\mu B-2 q \phi) \sin 2 \theta \simeq 0, \\
\Delta \theta & =\oint_{\text {orbit }} \frac{d z}{v_{\|}}\left[c \frac{\partial \phi}{\partial \psi}+\frac{c}{q} \hat{\kappa}_{\psi}(2 \epsilon-\mu B-2 q \phi)\right] \\
& \simeq \oint_{\text {orbit }} \frac{d z}{v_{\|}}\left(c \frac{\partial \phi}{\partial \psi}\right) .
\end{aligned}
$$

Here the zeroth-order orbit is that neglecting $\nabla B$ drifts compared with $\boldsymbol{E} \times \boldsymbol{B}$ drifts [i.e., orbit of $\mathcal{O}(1)$ in Eq. (10)].

Now we adopt a new coordinate system $(\psi, \bar{\theta}, \ell)$ to derive the neoclassical resonant diffusion applicable to the anchor mirror cell and transition mirror cells in GAMMA10. In this coordinate system $\psi=$ const on the same magnetic-flux tube, and an axis $\ell$ is taken as the closed drift orbit of the resonant ion with $\epsilon, \mu$, as shown in Fig. 3. We take $(\psi, \bar{\theta}, \ell)=(\psi, \theta, z)$ at the starting point of an ion under consideration, so that $\psi$ and $\bar{\theta}$ are constant along the zero-order orbit of a resonant ion with $\epsilon, \mu$. Therefore, the resonant condition in this coordinate is as follows:

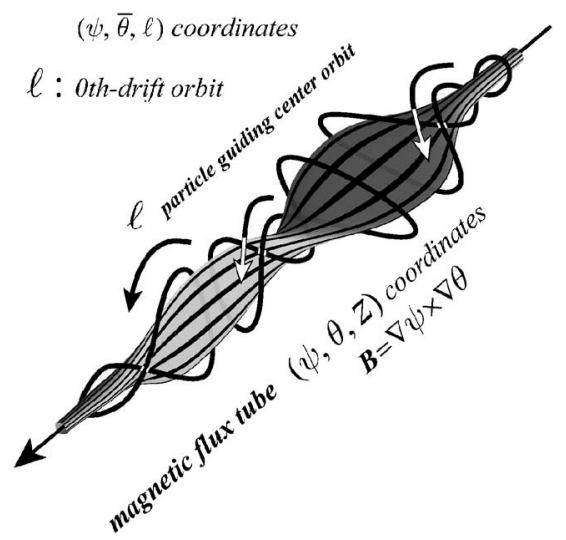

FIG. 3. Schematic diagram of the orbits of resonant ions. The magnetic-flux tube in the nonaxisymmetric region of GAMMA10 is shown with the magnetic-field lines shown as black stripes. Particle orbits are shown as solid closed curves, where a resonant ion started at some spatial point and comes back again at the same initial point after at least two axial bounce motions.

$$
\Delta \bar{\theta}\left(\psi, \epsilon_{k}, \mu\right)=0,
$$

where $\epsilon_{k}$ is a resonant energy which is a function of $\psi$ and $\mu$, but independent of $\bar{\theta}$ because the electrostatic potential $\phi$ is assumed to be a function of $\psi$ only. Using the Taylor expansion, ions near the resonant energy satisfy

$$
\Delta \bar{\theta}\left(\psi, \epsilon_{k}+\Delta \epsilon, \mu\right) \simeq \bar{\theta}_{\epsilon}^{\prime} \Delta \epsilon+\cdots,
$$

where $\bar{\theta}_{\epsilon}^{\prime} \equiv \partial \Delta \bar{\theta}\left(\psi, \epsilon_{k}, \mu\right) / \partial \epsilon$.

The drift kinetic equation for ion distribution function $f$ in the steady state is described as

$$
\dot{\psi} \frac{\partial f}{\partial \psi}+\dot{\bar{\theta}} \frac{\partial f}{\partial \bar{\theta}}+\dot{\ell} \frac{\partial f}{\partial \ell}=-\nu\left(f-f_{M}\right),
$$

where $\nu$ is the effective collision frequency, $f_{M}$ is a local Maxwellian defined as $f_{M}=n(\psi)\left[m_{i} / 2 \pi T_{i}(\psi)\right]^{3 / 2} \exp [-\epsilon$ $\left.-q \phi(\psi) / T_{i}(\psi)\right]$, and the dot symbol means the derivative with respect to time $t$. Equation (9) means that the distribution is constant along the ion drift orbit in the steady state.

We adopt the following ordering:

$$
\frac{\dot{\ell}}{\ell} \sim \mathcal{O}(1), \quad \frac{\dot{\psi}}{\psi} \sim \mathcal{O}(\varepsilon), \quad \dot{\bar{\theta}} \sim \mathcal{O}(\varepsilon), \quad \nu \tau_{\|} \sim \mathcal{O}(\varepsilon),
$$

where $\tau_{\|}$is an ion axial bounce time (i.e., time of axial one round trip), and $\varepsilon$ is a small expansion parameter. According to the above ordering, Eq. (9) reduces to $\dot{\ell}(\partial f / \partial \ell)=0$ in the zeroth order of $\varepsilon$ so that the distribution function $f$ is constant along the drift orbit without $\nabla B$ drifts and collisions, i.e., $f=f(\psi, \bar{\theta}, \epsilon, \mu)$, independent of the coordinate $\ell$.

The first-order equation in $\varepsilon$ of Eq. (9) is

$$
\dot{\psi} \frac{\partial f}{\partial \psi}+\dot{\bar{\theta}} \frac{\partial f}{\partial \bar{\theta}}=-\nu\left(f-f_{M}\right) \text {. }
$$

Bouncing averaging Eq. (11) along zeroth-order drift orbit $\ell$ we have 


$$
\langle\dot{\psi}\rangle \frac{\partial f}{\partial \psi}+\langle\dot{\bar{\theta}}\rangle \frac{\partial f}{\partial \bar{\theta}}=-\langle\nu\rangle\left(f-f_{M}\right) .
$$

Here,

$$
\begin{aligned}
& \langle\dot{\psi}\rangle=\frac{1}{\tau_{\|}} \oint \frac{d \ell}{\dot{\ell}} \dot{\psi}, \quad\langle\dot{\bar{\theta}}\rangle=\frac{1}{\tau_{\|}} \oint \frac{d \ell}{\dot{\varphi}} \dot{\bar{\theta}} \simeq \frac{\bar{\theta}_{\epsilon}^{\prime} \Delta \epsilon}{\tau_{\|}}, \\
& \langle\nu\rangle=\frac{1}{\tau_{\|}} \oint \frac{d \ell}{\dot{\ell}} \nu .
\end{aligned}
$$

During ion bounce motion along its guiding center zeroth orbit, ions feel $\nabla B$ drifts and collisions so that the distribution function changes from the Maxwellian distribution as $f=f_{M}(\psi, \epsilon, \mu)+\delta f(\psi, \bar{\theta}, \epsilon, \mu)$,

$$
\langle\dot{\psi}\rangle \frac{\partial f_{M}}{\partial \psi}+\frac{\bar{\theta}_{\epsilon}^{\prime}\left(\epsilon-\epsilon_{k}\right)}{\tau_{\|}} \frac{\partial \delta f}{\partial \bar{\theta}}=-\langle\nu\rangle \delta f .
$$

In the GAMMA10 tandem mirror with the usual quadrupole magnetic field $\langle\dot{\psi}\rangle$ is written as

$$
\langle\dot{\psi}\rangle=\frac{a(\psi, \epsilon, \mu)}{\tau_{\|}} \sin 2 \bar{\theta} .
$$

Therefore Eq. (14) is written as

$$
\frac{a \sin 2 \bar{\theta}}{\tau_{\|}} \frac{\partial f_{M}}{\partial \psi}+\frac{\bar{\theta}_{\epsilon}^{\prime}\left(\epsilon-\epsilon_{k}\right)}{\tau_{\|}} \frac{\partial \delta f}{\partial \bar{\theta}}=-\langle\nu\rangle \delta f .
$$

Fourier expansion of the distribution function $\delta f$ $=\Sigma_{m} \delta f_{m} \exp (i m \bar{\theta})$ we obtain

$$
\delta f=\frac{a}{2} \frac{\partial f_{M}}{\partial \psi}\left[\frac{\exp (2 i \bar{\theta})}{2 \bar{\theta}_{\epsilon}^{\prime}\left(\epsilon-\epsilon_{k}\right)-\tau_{\|}\langle\nu\rangle i}+\frac{\exp (-2 i \bar{\theta})}{2 \bar{\theta}_{\epsilon}^{\prime}\left(\epsilon-\epsilon_{k}\right)+\tau_{\|}\langle\nu\rangle i}\right] .
$$

In order to calculate the ion radial flux, we define the continuity equation of the total ion number $N$ in a tandem mirror as

$$
\begin{aligned}
-\frac{d N}{d t} & =\iiint \frac{d \psi d \theta d z}{B} \iint \frac{4 \pi B d \epsilon d \mu}{m_{i}^{2}\left|v_{\|}\right|} \nabla \cdot \boldsymbol{\Gamma} \\
& =\iint d \psi d \theta \iint \frac{4 \pi d \epsilon d \mu}{m_{i}^{2}} \int \frac{d z}{\left|v_{\|}\right|} \nabla \cdot \boldsymbol{\Gamma} .
\end{aligned}
$$

Here,

$$
\boldsymbol{\Gamma} \cdot \nabla \psi=\sum_{k=1,3,5, \ldots} \delta f \dot{\psi},
$$

where $\Sigma_{k=1,3,5, \ldots}$ in Eq. (19) means the sum on a resonant energy of $\epsilon_{k}$. Transformation of Eq. (18) represented by $(\psi, \theta, z)$ to the coordinates $(\psi, \bar{\theta}, \ell)$ is given by

$$
\begin{aligned}
-\frac{d N}{d t}= & \iint d \psi d \bar{\theta} \iint \frac{4 \pi d \epsilon d \mu}{m_{i}^{2}} \\
& \times \int \frac{B d \ell}{(\nabla \psi \cdot \nabla \bar{\theta} \times \nabla \ell)\left|v_{\|}\right|} \nabla \cdot \boldsymbol{\Gamma} .
\end{aligned}
$$

Recalling that $\ell$ is taken along the zeroth-order guiding center orbit of the resonant ion, the transformation to $\bar{\theta}$, where $\dot{\bar{\theta}}=0$ along the zeroth-order orbit, is given by

$$
\bar{\theta}=\theta-c \frac{\partial \phi(\psi)}{\partial \psi} t \Rightarrow \nabla \bar{\theta}=\nabla \theta-\nabla \psi\left[c \frac{\partial^{2} \phi(\psi)}{\partial \psi^{2}} t\right] .
$$

So,

$$
\begin{aligned}
(\nabla \psi \cdot \nabla \bar{\theta} \times \nabla \ell)\left|v_{\|}\right| & =\nabla \psi \times \nabla \theta \cdot \nabla \ell\left|v_{\|}\right| \\
& =\boldsymbol{B} \cdot \nabla \ell\left|v_{\|}\right|=B \frac{d \ell}{d z}\left|v_{\|}\right|=B|\dot{\ell}| .
\end{aligned}
$$

Therefore,

$$
\begin{aligned}
-\frac{d N}{d t} & =\iint d \psi d \bar{\theta} \iint \frac{4 \pi d \epsilon d \mu}{m_{i}^{2}} \oint \frac{d \ell}{2 \dot{\ell}} \nabla \cdot \boldsymbol{\Gamma}(\psi, \bar{\theta}, \epsilon, \mu)=\iint d \psi d \bar{\theta} \iint \frac{4 \pi d \epsilon d \mu}{m_{i}^{2}} \oint \frac{d \ell}{2 \dot{\ell}} \boldsymbol{B} \cdot \nabla \ell \frac{\partial}{\partial \psi}\left[\frac{\boldsymbol{\Gamma}(\psi, \bar{\theta}, \epsilon, \mu) \cdot \nabla \psi}{\boldsymbol{B} \cdot \nabla \ell}\right] \\
& =\sum_{k=1,3,5, \ldots} \int_{0}^{\psi} d \psi \frac{\partial}{\partial \psi}\left(\int_{0}^{2 \pi} d \bar{\theta} \iint \frac{4 \pi d \epsilon d \mu}{m_{i}^{2}} \oint \frac{d \ell}{2 \dot{\ell}} \delta f \dot{\psi}\right)=\sum_{k=1,3,5, \ldots} \int_{0}^{2 \pi} d \bar{\theta} \iint \frac{4 \pi d \epsilon d \mu}{m_{i}^{2}} \delta f \frac{a \sin 2 \bar{\theta}}{2} .
\end{aligned}
$$

Here Eqs. (13), (15), and (19) are used to obtain Eq. (23).

With Eq. (23) the radial particle flux $\left\langle\Gamma_{\psi}\right\rangle$ in a nonaxi- symmetric mirror cell, averaged over the magnetic-flux surface, is defined as 


$$
\left\langle\Gamma_{\psi}\right\rangle \equiv \frac{1}{2 \pi L_{m}} \sum_{k} \int_{0}^{2 \pi} d \bar{\theta} \iint \frac{4 \pi d \epsilon d \mu}{m_{i}^{2}} \delta f \frac{a \sin 2 \bar{\theta}}{2} .
$$

Here $L_{m}$ is an axial length of the nonaxisymmetric mirror cell, and the different resonances are assumed nonoverlapping and contributing independently to the flux. Taking the limit $\nu \tau_{\|} \rightarrow+0$, the radial flux is obtained as

$$
\left\langle\Gamma_{\psi}\right\rangle=-\frac{\pi^{2}}{2 m_{i}^{2} L_{m}} \sum_{k} \int d \mu\left[\frac{a^{2}(\psi, \epsilon, \mu)}{\left|\bar{\theta}_{\epsilon}^{\prime}\right|} \frac{\partial f_{M}}{\partial \psi}\right]_{\epsilon=\epsilon_{k}(\psi, \mu)} .
$$

Here,

$$
\frac{\partial f_{M}}{\partial \psi}=\left(\frac{1}{n} \frac{\partial n}{\partial \psi}-\frac{3}{2 T_{i}} \frac{\partial T_{i}}{\partial \psi}+\frac{\epsilon_{k}-q \phi}{T_{i}^{2}} \frac{\partial T_{i}}{\partial \psi}+\frac{q}{T_{i}} \frac{\partial \phi}{\partial \psi}\right) f_{M} .
$$

Equation (25) is similar to Eq. (5) of Myra and Catto. ${ }^{23}$ Both equations will lead to the same result under the problem such that a tandem mirror has an axisymmetric central cell with a nonaxisymmetric mirror located at both ends of the device. However, Eq. (25) can be applied to the ions trapped in the transition region of GAMMA10, as shown in Fig. 2, where the magnetic-flux tube is fully nonaxisymmetric and does not have an axial symmetry, in the case of which the $\nabla B$ drifts should be evaluated along the closed drift orbits of resonant particles with $(\psi, \bar{\theta}, \ell)$ coordinates in the transition of GAMMA10.

The diffusion coefficient of neoclassical resonant plateau transport is defined as

$$
D_{j}=\left(\frac{m_{i}}{2 \pi T_{i}}\right)^{3 / 2} \sum_{k} \int d \mu A_{k}\left(\frac{W_{k}}{T_{i}}\right)^{j} \exp \left(-\frac{W_{k}}{T_{i}}\right),
$$

where $W_{k}$ and $A_{k}$ are described as

$$
W_{k}=\epsilon_{k}(\psi, \mu)-q \phi(\psi), \quad A_{k}=\frac{\pi^{2}}{2 m_{i}^{2} L_{m}} \frac{a^{2}\left(\psi, \epsilon_{k}, \mu\right)}{\left|\bar{\theta}_{\epsilon}^{\prime}\left(\psi, \epsilon_{k}, \mu\right)\right|} .
$$

The radial flux of neoclassical resonant diffusion is written with the diffusion coefficient in Eq. (27) as

$$
\left\langle\Gamma_{\psi}\right\rangle=-\left[D_{0} \frac{\partial n}{\partial \psi}+\frac{n}{T_{i}}\left(D_{1}-\frac{3}{2} D_{0}\right) \frac{\partial T_{i}}{\partial \psi}+\frac{n q}{T_{i}} D_{0} \frac{\partial \phi}{\partial \psi}\right] .
$$

To determine the flux of Eq. (29), the diffusion coefficients in Eq. (27) are required. The actual orbit calculation is carried out in the GAMMA10 magnetic configuration to obtain the neoclassical diffusion.

\section{APPLICATION TO THE GAMMA10 TANDEM MIRROR}

The analytical formulation obtained in Sec. III to evaluate the neoclassical resonant plateau transport in a nonaxisymmetric magnetic field is applied to the GAMMA10 tandem mirror. In this section the numerical calculation is carried out for the neoclassical resonant transport in the nonaxisymmetric magnetic-field (anchor) mirror cell and transition mirror cells in GAMMA10.

\section{A. Resonant energy}

We assume that the electrostatic potential $\phi$ is constant along the magnetic-field line and its radial profile is described as

$$
\phi(\psi)=\phi_{0}\left(1-\frac{\psi}{\psi_{0}}\right) .
$$

Here $\psi_{0}=\frac{1}{2} L_{\phi}^{2} B(z=0)$, and $L_{\phi}=20 \mathrm{~cm}$.

First we examine the neoclassical resonant transport for each nonaxisymmetric mirror cell of GAMMA10. The quantities $a, \epsilon_{k}$, and $\partial \Delta \bar{\theta} / \partial \epsilon_{k}$ in Eq. (28) are obtained by Eq. (6) as follows. The radial step size $a$ is calculated by

$$
\begin{aligned}
\Delta \psi & =-\oint \frac{d \ell}{\dot{\ell}}\left\{\frac{c}{q} \hat{\kappa}_{\theta} \psi\left[2 \epsilon_{k}-\mu B(z)-2 q \phi(\psi)\right] \sin 2 \theta\right\} \\
& =a_{k}\left(\psi, \epsilon_{k}, \mu\right) \sin 2 \bar{\theta}_{p},
\end{aligned}
$$

where $\bar{\theta}_{p}$ is the coordinate determined by each nonaxisymmetric mirror. The resonance condition is described as

$$
\begin{aligned}
& \Delta \bar{\theta}\left(\psi, \epsilon_{k}, \mu\right)=\oint \frac{d z}{v_{\|}\left(\psi, z, \epsilon_{k}, \mu\right)}\left[c \frac{\partial \phi(\psi)}{\partial \psi}\right]-k \pi=0, \\
& k=1,3,5, \ldots,
\end{aligned}
$$

where $v_{\|}\left(\psi, z, \epsilon_{k}, \mu\right)=\left\{2 / m_{i}\left[\epsilon_{k}-\mu B(z)-q \phi(\psi)\right]\right\}^{1 / 2}$ with hydrogen ion mass $m_{i}$ and charge $q$. The resonant energy $\epsilon_{k}$ is obtained by Eq. (32) as a function of $\psi$ and $\mu$, and the axis $\ell$ is also determined by Eq. (32). Expression (32) leads to the following energy derivative of $\Delta \bar{\theta}\left(\psi, \epsilon_{k}, \mu\right)$ :

$$
\bar{\theta}_{\epsilon}^{\prime}\left(\psi, \epsilon_{k}, \mu\right) \simeq-\frac{1}{m_{i}} \oint \frac{d z}{v_{\|}^{3 / 2}\left(\psi, z, \epsilon_{k}, \mu\right)}\left[c \frac{\partial \phi(\psi)}{\partial \psi}\right] .
$$

Figure 4 shows the resonance lines of $k=1$ in the velocity $(\epsilon, \mu)$ by a bold curve, where $\phi_{0}=300 \mathrm{~V}$ and $\epsilon_{0}$ $=100 \mathrm{eV}$. The quantities $B_{M}, B_{A}$, and $B_{T}$ are the magnetic fields at the mirror throat of the anchor mirror cell, at the anchor mirror cell midplane, and at the transition mirror cell midplane, respectively. The vertical axes in Figs. 4(a)-4(c) are the kinetic energies and the horizontal axis is the kinetic energy perpendicular to the magnetic-field line. The arrows in each figure are plotted for the purpose of the relation to Figs. 5 and 6, which is described later in this section. The resonant ions trapped in the anchor mirror cell in Fig. 4(a) do not depend on $\mu$ so much, because the ion bounce time $\tau_{\|}$is a function of $\mu$ but not of $\epsilon$ in the parabolic magnetic axial profile such as the anchor mirror cell. The resonant ions trapped in the transition mirror cell in Figs. 4(b) and 4(c) depend on $\epsilon-\mu B_{T}$ for deeply trapped ions, where the gradient of the magnetic-field axial profile $(d B / d z)$ is approximately constant around the ion turning point, while shallow trapped ions has a different dependence on $\mu$ from the deeply trapped resonant ions due to the change of magneticfield axial profiles around the ion turning point.

Figures 5(a)-5(c) plot the radial shift $\Delta \psi$ of the resonant ions per one-bounce axial motion, where the radial shifts along the direction of the lines with arrow correspond to the 

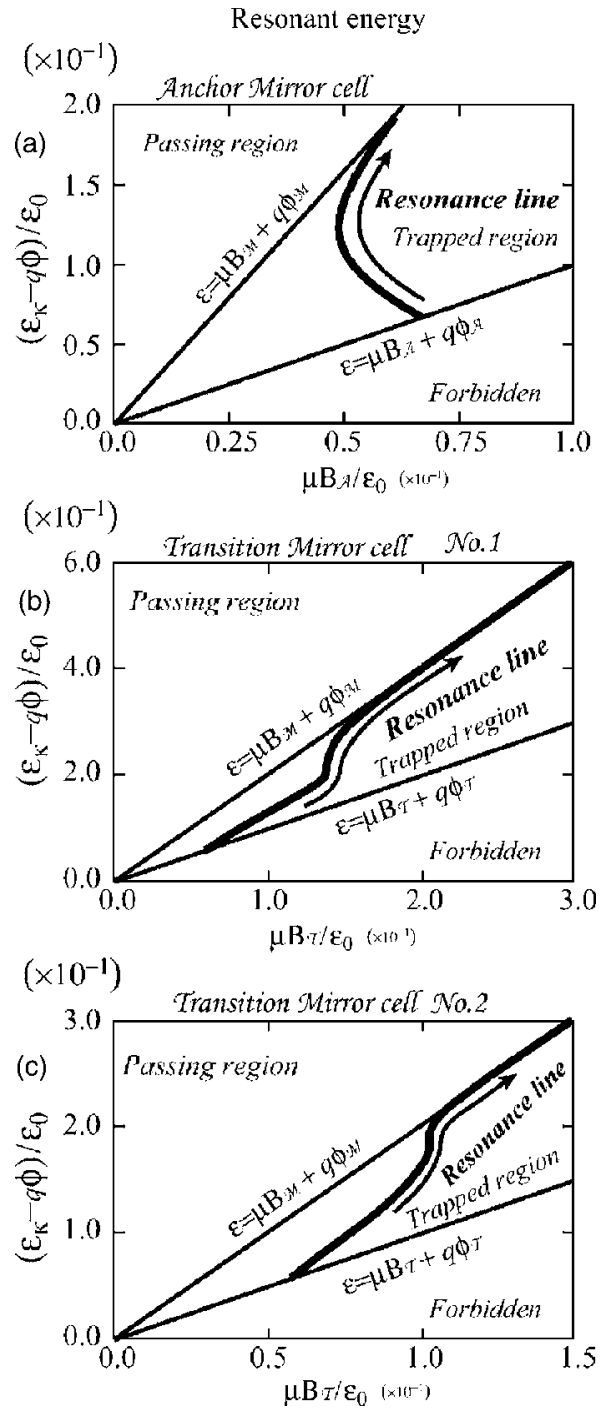

FIG. 4. The regions of resonant ions in the velocity space $(\epsilon, \mu)$ are plotted. (a) is the resonant ions trapped in the "Anchor Mirror Cell." (b) is the resonant ions trapped in "Transition Mirror Cell No. 1." (c) is the resonant ions trapped in "Transition Mirror Cell No. 2."

resonance lines along the lines with arrows in Figs. 4(a) $-4(\mathrm{c})$, respectively. The radial shift $\Delta \psi$ is calculated by the drift motion of resonant ions [Eq. (31)]. That is, resonant ions which started at $\left(\psi_{\text {ini }}, \bar{\theta}_{\text {ini }}, z_{\text {ini }}\right)$ come back again at the same spatial point $\left(\psi_{\text {ini }}+\Delta \psi, \bar{\theta}_{\text {ini }}, z_{\text {ini }}\right)$ after the one axial round trip in a mirror cell. The amount of the radial shift $\Delta \psi$ is proportional to $\sin \left(2 \bar{\theta}_{\text {ini }}+\alpha\right)$, as shown in Eq. (31), so that $\Delta \psi\left(\bar{\theta}_{\text {ini }}\right)$ is expanded in the Fourier series of $\bar{\theta}_{\text {ini. }}$. Figure 5 shows the amplitude of $\Delta \psi$ proportional to $\sin \left(2 \bar{\theta}_{\text {ini }}+\alpha\right)$, where $\alpha$ is constant. It is found that the shallow trapped resonant ions has a larger radial shift than the deeply trapped resonant ions in a mirror cell.

Figure 6 plots the magnitude of $\left|\partial \Delta \bar{\theta} / \partial \epsilon_{k}\right|$, where the radial shifts along the direction of the lines with arrow correspond to the resonance lines along the lines with arrows in Figs. 4(a)-4(c), respectively. As seen in Eq. (25) the radial particle flux is inversely proportional to $\left|\partial \Delta \bar{\theta} / \partial \epsilon_{k}\right|$. So Fig. 6
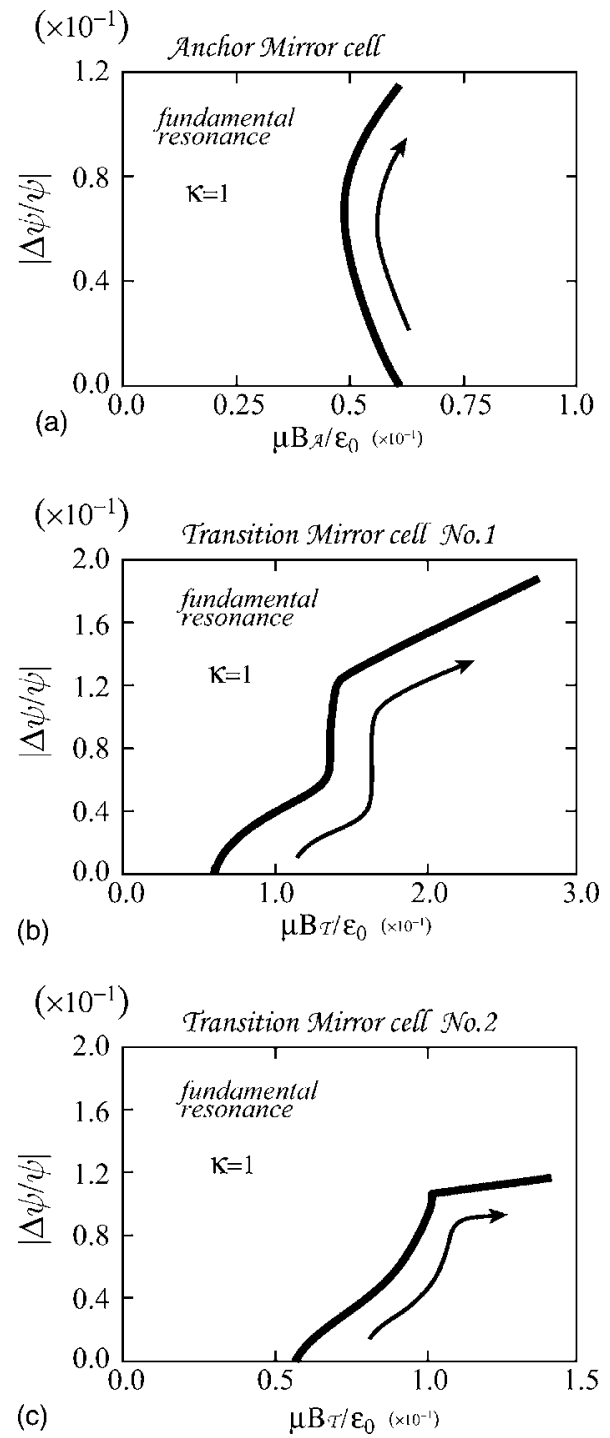

FIG. 5. The regions of resonant ions in the velocity space and radial shift $\Delta \psi$ of resonant ions per one axial bounce. (a) is a radial shift of the resonant ions trapped in the "Anchor Mirror Cell." (b) is a radial shift of the resonant ions trapped in "Transition Mirror Cell No. 1." (c) is a radial shift of the resonant ions trapped in "Transition Mirror Cell No. 2."

shows that the deeply trapped resonant ions contribute to the radial transport rather than the shallow trapped resonant ions in each mirror cell.

\section{B. Diffusion coefficients}

The diffusion coefficients given in Eq. (27) are determined as follows:

$$
\begin{aligned}
& D_{j}=\sum_{k} \int \frac{d \mu}{\mu_{0}}\left(\frac{W_{k}}{T_{i}}\right)^{j} \tilde{D}_{k}, \\
& \tilde{D}_{k}=\mu_{0}\left(\frac{m_{i}}{2 \pi T_{i}}\right)^{3 / 2} \frac{\pi^{2}}{2 m_{i}^{2} L_{m}} \frac{a^{2}\left(r, \mu, \epsilon_{k}\right)}{\left|\bar{\theta}_{\epsilon}^{\prime}\left(r, \mu, \epsilon_{k}\right)\right|} \exp \left(-\frac{\epsilon_{k}-q \phi}{T_{i}}\right) .
\end{aligned}
$$

Here $\mu_{0}=\epsilon_{0} / B_{\mathrm{cc}}$ with magnetic field $B_{\mathrm{cc}}$ at the central cell midplane of GAMMA10 under consideration. Integrating 

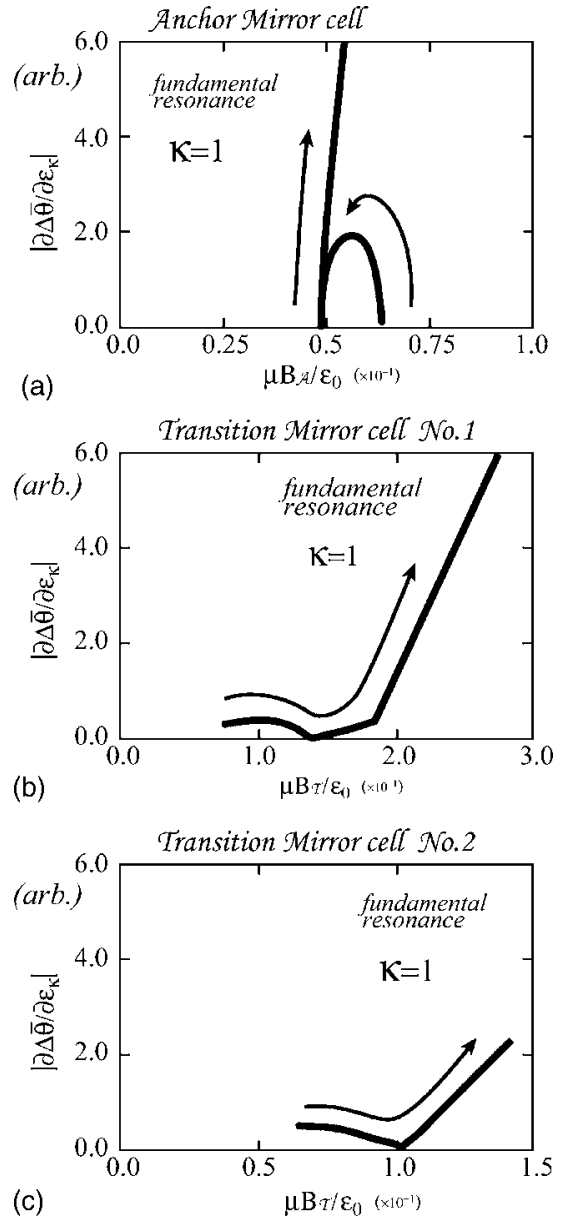

FIG. 6. The regions of resonant ions in the velocity space and a quantity $\left|\partial \Delta \bar{\theta} / \partial \epsilon_{k}\right|$ of resonant ions per one axial bounce. (a) is $\left|\partial \Delta \bar{\theta} / \partial \epsilon_{k}\right|$ of the resonant ions trapped in the "Anchor Mirror Cell." (b) is $\left|\partial \Delta \bar{\theta} / \partial \epsilon_{k}\right|$ of the resonant ions trapped in "Transition Mirror Cell No. 1." (c) is $\left|\partial \Delta \bar{\theta} / \partial \epsilon_{k}\right|$ of the resonant ions trapped in "Transition Mirror Cell No. 2."

Eq. (34) numerically, the magnitude of the diffusion coefficient is determined. Please note that Eq. (34) gives the diffusion coefficients resulting from the resonant ions trapped in a mirror cell with axial length of $L_{m}$.

Figure 7 shows the diffusion coefficient $D_{0}$ on the flux surface $\psi=\frac{1}{2}(1 \mathrm{~cm})^{2} B_{\mathrm{cc}}$ as a function of $\Delta \theta_{\mathrm{th}}$ in the case of $T_{i}=200 \mathrm{eV}$. The diffusion coefficient resulting from resonant ions trapped in the central cell is inserted in Fig. 7(d) as a reference, ${ }^{21}$ where the resonant ions pass from the mirror throat around $z \simeq-750 \mathrm{~cm}$ to another mirror throat around $z \simeq 750 \mathrm{~cm}$ in Fig. 1 . Contributions to $D_{0}$ from different resonances $(k=1-19)$ are shown together. Here $\Delta \theta_{\text {th }}$ is an azimuthal displacement by $\boldsymbol{E} \times \boldsymbol{B}$ drifts per half-period of ion bounce motion, defined as

$$
\Delta \theta_{\mathrm{th}}=\frac{L_{\mathrm{sys}}}{\left(T_{i} / m_{i}\right)^{1 / 2}} c \frac{\partial \phi}{\partial \psi},
$$

where $L_{\mathrm{sys}}$ is the axial length of the GAMMA10 tandem mirror [i.e., length from a plug at the left side $(z$ $=-960 \mathrm{~cm})$ to another plug at the right side $(z=960 \mathrm{~cm})]$. The coefficient $D_{1}$ has the same dependences on $k$ and $\Delta \theta_{\text {th }}$ that are essentially similar to those of $D_{0}$ except that typi- cally $D_{1} \sim 3 D_{0}$. For large $k$, the resonant diffusion is small because the large rotation in the nonaxisymmetry region smoothes out the nonaxisymmetry. The resonant diffusion coefficients $D_{j}$ in Eq. (34) at an ion temperature are obtained by summing up numerical integration of $\widetilde{D}_{k}$ over $\epsilon_{k}$ for each $k$. These diffusion coefficients are plotted by solid lines in Fig. 7.

Figure 8 shows the diffusion coefficient $D_{0}$ on the flux surface $\psi=\frac{1}{2}(1 \mathrm{~cm})^{2} B_{\mathrm{cc}}$ for several ion temperatures in each mirror cell. The diffusion coefficient resulting from resonant ions trapped in the central cell is inserted in Fig. 8(d) as a reference. ${ }^{21}$ It is found that $D_{0}$ decreases with $T_{i}$ due to the decrease of the population of resonant ions except for the central cell in Fig. 8(d), where the population of resonant ions is proportional to $\epsilon_{k} / T_{i}^{3 / 2} \exp \left\{-\epsilon_{k} / T_{i}\right\} d \epsilon_{k}^{1 / 2}$. Figure 8(a) shows the diffusion coefficients $D_{0}$ resulting from resonant ions trapped in the anchor mirror cell, which is similar to the case of transition mirror cell No. 1 in Fig. 8(b) and transition mirror cell No. 2 in Fig. 8(c). The coefficient $D_{1}$ has almost the same dependences on $T_{i}$ and $\Delta \theta_{\text {th }}$ that are essentially similar to those of $D_{0}$ except that typically $D_{1} \sim 3 D_{0}$ for $T_{i}$ $=200 \mathrm{eV}$, and $D_{1} \sim 1 / 2 D_{0}$ for $T_{i}=2 \mathrm{keV}$ in each mirror cell.

The dependence of diffusion coefficients on the ion temperature of the anchor mirror cell and transition mirror cells is contrary for the case of the central mirror cell because the resonant energies $\epsilon_{k}$ in the anchor and transition mirror cells are much smaller than that in the central mirror cell.

\section{Ion confinement time}

Finally we evaluate the confinement time $\tau_{\perp}$ attributed to the neoclassical resonant plateau transport in each mirror cell in GAMMA10. The ion radial flux given by Eq. (29) leads to the loss of total number $N$ of ions in GAMMA10, where

$$
N=\iiint \frac{n d \psi d \theta d z}{B}
$$

With Eqs. (23) and (24),

$$
\begin{aligned}
-\frac{d N}{d t}= & 2 \pi L_{\mathrm{cc}}\left\langle\Gamma_{\psi}^{\mathrm{cc}}\right\rangle+4 \pi L_{A}\left\langle\Gamma_{\psi}^{A}\right\rangle+4 \pi L_{\mathrm{tr} 1}\left\langle\Gamma_{\psi}^{\mathrm{tr} 1}\right\rangle \\
& +4 \pi L_{\mathrm{tr} 2}\left\langle\Gamma_{\psi}^{\mathrm{tr} 2}\right\rangle .
\end{aligned}
$$

Here $L_{\mathrm{cc}}, L_{A}, L_{\mathrm{tr} 1}$, are $L_{\mathrm{tr} 2}$ are the axial lengths of the central cell, anchor mirror cell, transition mirror cell No. 1, and transition mirror cell No. 2, respectively. The particle fluxes $\left\langle\Gamma_{\psi}^{\mathrm{cc}}\right\rangle,\left\langle\Gamma_{\psi}^{A}\right\rangle,\left\langle\Gamma_{\psi}^{\mathrm{tr} 1}\right\rangle$, and $\left\langle\Gamma_{\psi}^{\mathrm{tr} 2}\right\rangle$ are the neoclassical particle fluxes, which are calculated by Eq. (29) on the flux surface $\psi=\psi_{\text {core }}$ with the relation of $D_{j} \propto \psi$ radially in Eq. (34) by using the diffusion coefficient $D_{0}$ shown in Fig. 8 and the coefficient $D_{1}$. The radius $\psi_{\text {core }}$ is the core region of plasma in the radial direction, which is given as $\psi_{\text {core }}$ $=\frac{1}{2}(10 \mathrm{~cm})^{2} B_{\mathrm{cc}}$. We assume that the axial and azimuthal distributions of ion density and temperature are uniform, and so the radial ion density $n(\psi)$ is given as 

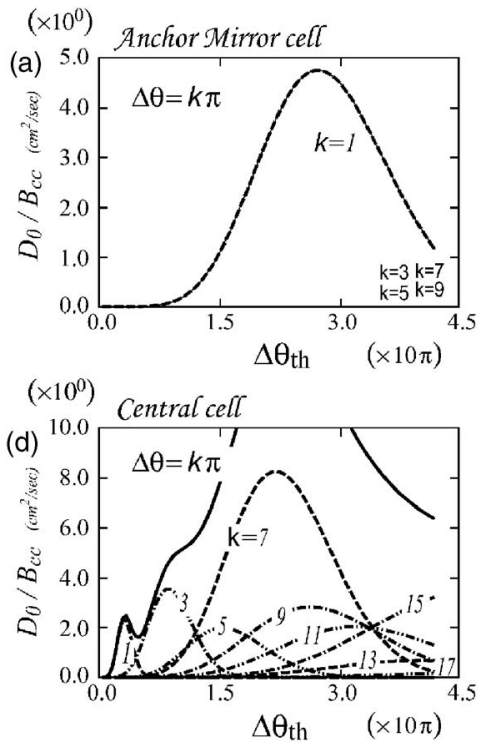

$$
n(\psi)=n_{0} \exp \left(-\frac{\psi}{\psi_{n}}\right),
$$

and the ion temperature $T_{i}(\psi)$ is given as

$$
T_{i}(\psi)=T_{i 0} \exp \left(-\frac{\psi}{\psi_{T}}\right),
$$

where $\psi_{n}=\frac{1}{2}(20 \mathrm{~cm})^{2} B_{\mathrm{cc}}$ and $\psi_{T}=\frac{1}{2}(20 \mathrm{~cm})^{2} B_{\mathrm{cc}}$ so that $\psi_{\text {core }} / \psi_{n}=\psi_{\text {core }} / \psi_{T}=\frac{1}{4}$.

In order to find which mirror cell is responsible for the ion radial confinement, the radial confinement times $\tau_{\perp}$ resulting from the radial diffusion in each mirror cell are defined as

$$
\begin{gathered}
\tau_{\perp}^{\mathrm{tr} 1} \equiv \frac{N}{2 \pi L_{\mathrm{tr} 1}\left\langle\Gamma_{\psi}^{\mathrm{tr} 1}\right\rangle}, \quad \tau_{\perp}^{A} \equiv \frac{N}{2 \pi L_{A}\left\langle\Gamma_{\psi}^{A}\right\rangle}, \\
\tau_{\perp}^{\mathrm{tr} 2} \equiv \frac{N}{2 \pi L_{\mathrm{tr} 2}\left\langle\Gamma_{\psi}^{\mathrm{tr} 2}\right\rangle}, \quad \tau_{\perp}^{\mathrm{cc}} \equiv \frac{N}{2 \pi L_{\mathrm{cc}}\left\langle\Gamma_{\psi}^{\mathrm{cc}}\right\rangle},
\end{gathered}
$$

where $N$ is the total ion number confined within the core region $\psi \leqslant \psi$ core of the tandem mirror given as

$$
\begin{aligned}
N & =\int \frac{\mathrm{d} z}{B(z)} \int_{0}^{\psi_{\text {core }}} n(\psi) 2 \pi \mathrm{d} \psi \\
& \simeq \frac{\pi L_{\text {sys }} \psi_{n}}{B_{c c}} n_{0}\left[1-\exp \left(-\frac{\psi_{\text {core }}}{\psi_{n}}\right)\right] .
\end{aligned}
$$

Figure 9 plots the confinement time $\tau_{\perp}$ for various cases of ion temperatures $T_{i}\left(\psi_{\text {core }}\right)$ on the flux $\psi=\psi_{\text {core }}$ in each mirror cell as a function of radial electric field $\Delta \theta_{\text {th }}$. The confinement time resulting from resonant ions trapped in the central cell are inserted in Fig. 9(d) as a reference. ${ }^{21}$ The ion radial confinement times resulting from resonant ions trapped in the anchor mirror cell [Fig. 9(a)], in transition mirror cell No. 1 [Fig. 9(b)], and in transition mirror cell No. 2 [Fig. 9(c)] as well as that in the central cell [Fig. 9(d)] in the GAMMA10 are obtained as a function of radial electric field and ion temperature. The results made it clear that the neoclassical diffusions in the anchor mirror cell and transition mirror cells are sufficiently smaller than that in the central mirror cell. The resonant ions in the anchor mirror cell and transition mirror cells have a lower energy than that in the central cell because the anchor and transition mirror axial lengths are much shorter than that of the central cell. The amount of $\nabla B$ drift leading to the neoclassical resonant transport is small due to the low energy of resonant ions in the anchor cell and transition mirror cells, so that the neoclassical resonant transport in GAMMA10 is important mainly in the central cell.

\section{SUMMARY AND DISCUSSIONS}

The electrostatic potential is assumed to be $\phi=\phi(\psi)$ in this paper. That is, the axial dependence of $\phi$ is not taken into account in Sec. IV, though the analytically obtained Eq. (25) is applicable to the case of $\phi=\phi(\psi, z)$. The main reason for neglecting the axial variation of $\phi$ is that $\phi$ is desirable to be constant along $z$ in the nonaxisymmetric region experimentally, as shown in Fig. 1(d) in order to avoid the ions and electrons coming from the central cell from being reflected by the electrostatic potential in the nonaxisymmetric region.

Figure 5 shows that the radial shift of shallow trapped ions is larger than that of deeply trapped ions, while Fig. 6 reveals that more population of deeply trapped resonant ions exists than that of shallow trapped resonant ions. In other words, the resonant ions with a turning point close to the midplane of a mirror cell have a wider resonant region in the velocity space, and the resonant ions with a turning point close to the mirror throat have larger radial shifts. If there is an electrostatic potential of well-type, that is minimum at the midplane, in a mirror cell, the ions with higher energy have a turning point at each axial position. The higher-energy ions mean the lower population of Maxwellian ions so that neoclassical resonant transport is relatively small in the case of a well-type electrostatic potential. Hill-type electrostatic potential enhances the neoclassical resonant transport of ions coming from the central cell of GAMMA10 much more than that of ions trapped in each mirror cell. 
$\left(\times 10^{1}\right)$

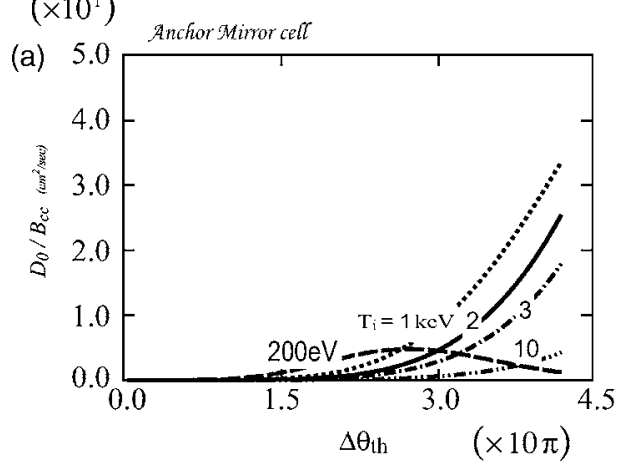

$\left(\times 10^{3}\right)$

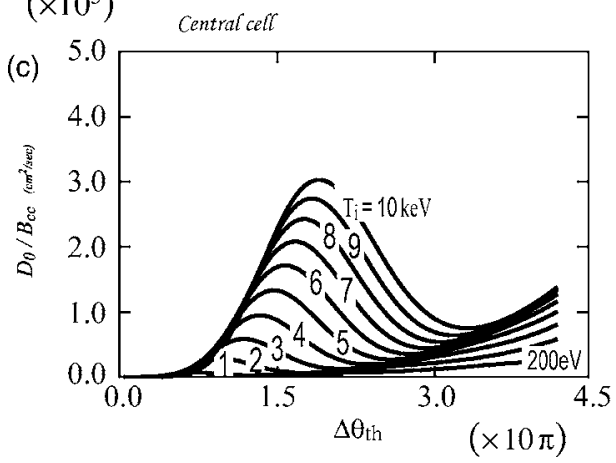

$\left(\times 10^{1}\right)$

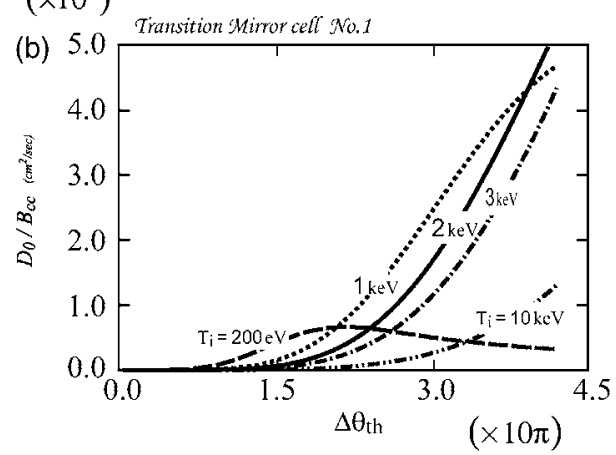

$\left(\times 10^{1}\right)$

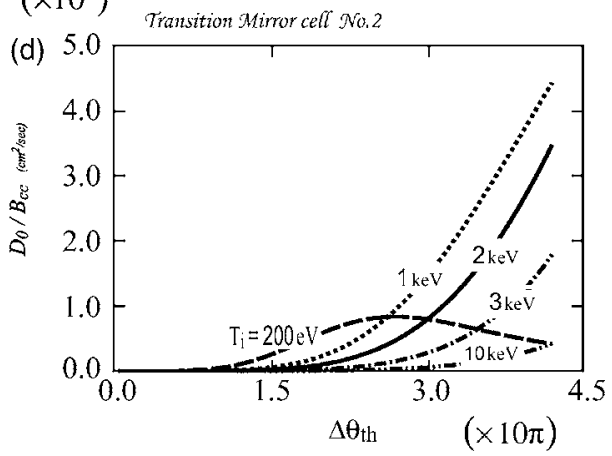

FIG. 8. Diffusion coefficient $D_{0}$ for various ion temperatures $T_{i}$. Here in the calculation of $\Delta \theta_{\text {th }}$ of horizontal axis, given in Eq. (35), $T_{i}=200 \mathrm{eV}$ is assumed. (a) is "Anchor Mirror Cell," (b) is "Transition Mirror Cell No. 1," (c) is "Transition Mirror Cell No. 2," and (d) is the "Central Cell," respectively.
As a summary calculations are made of the neoclassical resonant transport in the anchor mirror cell and transition mirror cell in the GAMMA10 tandem mirror. The diffusion coefficient and confinement time are evaluated. The results are summarized as follows.

The analytical formulation of neoclassical resonant plateau transport which is applied to an arbitrary magnetic mirror configuration is developed with the use of the coordination system $(\psi, \bar{\theta}, \ell)$. This formulation reduces to the conventional theory by Ryutov and Stupakov ${ }^{19,20}$ in the limit of a square-well magnetic field. The radial ion loss attributable to the neoclassical diffusion is evaluated by applying the theory of neoclassical diffusion to the nonaxisymmetric magnetic-field anchor mirror cell and transition mirror cell in GAMMA10. The ion radial diffusion and confinement time of the anchor mirror cell, transition mirror cell, and central mirror cell in GAMMA10 are obtained as a function of the radial electric field and ion temperature.

The results made it clear that the neoclassical diffusions resulting from the resonant ions trapped in the anchor mirror
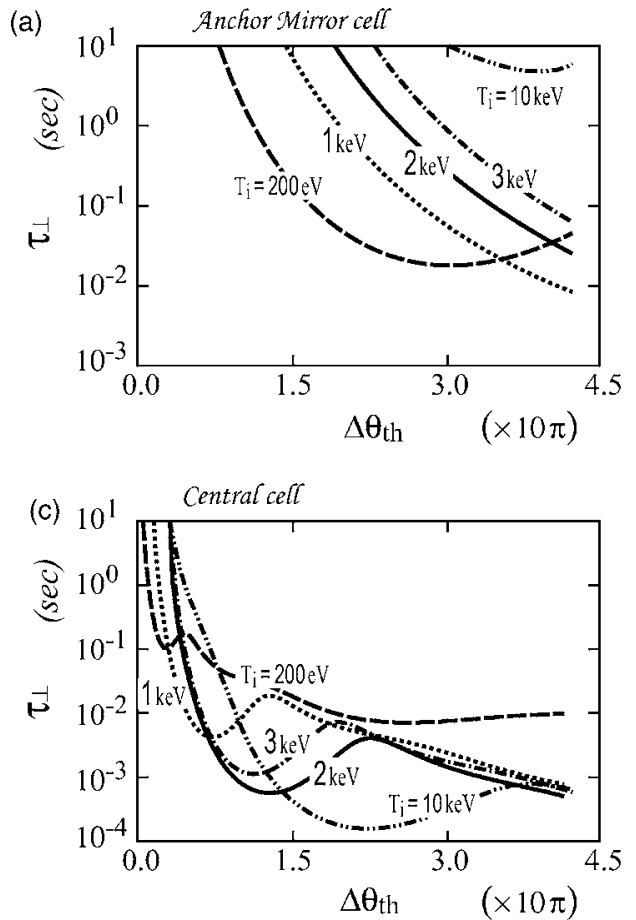
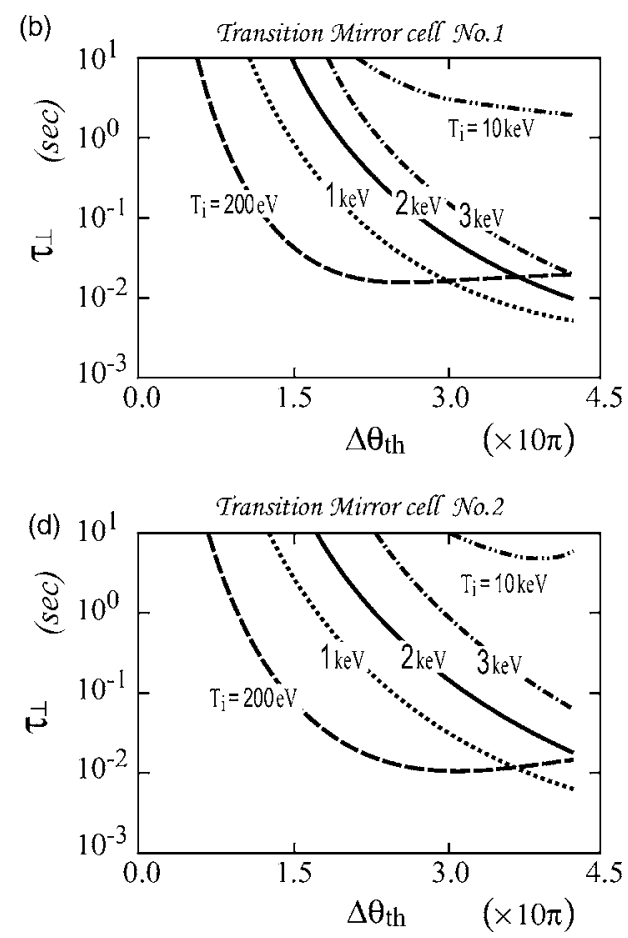

FIG. 9. Ion radial confinement time $\tau_{\perp}$. (a) is $\tau_{\perp}^{A}$ resulting from the radial transport of resonant ions trapped in the "Anchor Mirror Cell," (b) is $\tau_{\perp}^{\mathrm{tr} 1}$ from "Transition Mirror Cell No. 1," (c) is $\tau_{\perp}^{\mathrm{tr} 2}$ from "Transition Mirror Cell No. 2," and (d) is $\tau_{\perp}^{\mathrm{cc}}$ from the "Central Cell," respectively. 
cell and transition mirror cell are sufficiently smaller than those trapped in the central mirror cell, because the resonant ions trapped in the anchor mirror cell and transition mirror cell have a much lower energy than those trapped in the central cell.

${ }^{1}$ G. I. Dimov, V. V. Zakaidakov, and M. E. Kishinevskij, Fiz. Plazmy 2, 597 (1976) [Sov. J. Plasma Phys. 2, 326 (1976)].

${ }^{2}$ T. K. Fowler and B. G. Logan, Comments Plasma Phys. Controlled Fusion 2, 167 (1977).

${ }^{3}$ For example, see R. F. Post, Nucl. Fusion 27, 1579 (1987).

${ }^{4}$ A. J. Lichtenberg and M. A. Lieberman, Regular and Chaotic Dynamics (Springer, New York, 1992), Chap. 1.

${ }^{5}$ W. Horton and Y.-H. Ichikawa, Chaos and Structures in Nonlinear Plasmas (World Scientific, NJ, 2000), p. 125.

${ }^{6}$ Y.-H. Ichikawa, T. Kamimura, and C. F. F. Karney, Physica D 6, 233 (1983).

${ }^{7}$ T. B. Kaiser, W. M. Nevins, and L. D. Pearlstein, Phys. Fluids 26, 351 (1983).

${ }^{8}$ I. Katanuma, Y. Tatematsu, K. Ishii, T. Tamano, and K. Yatsu, J. Phys. Soc. Jpn. 69, 3244 (2000)

${ }^{9}$ L. S. Hall and B. McNamara, Phys. Fluids 18, 552 (1975).

${ }^{10}$ G. V. Stupakov, Fiz. Plazmy 5, 871 (1979) [Sov. J. Plasma Phys. 5, 486 (1979)].

${ }^{11}$ L. D. Pearlstein, T. B. Kaiser, and W. A. Newcomb, Phys. Fluids 24, 1326 (1981); see also 24, 1326(E) (1981).

${ }^{12}$ B. Lane, R. S. Post, and J. Kesner, Nucl. Fusion 27, 277 (1987)

${ }^{13}$ V. P. Pastukhov and A. Yu. Sokolov, Fiz. Plazmy 17, 1043 (1991) [Sov. J. Plasma Phys. 17, 603 (1991)].

${ }^{14}$ I. Katanuma, T. Ito, H. Saimaru, Y. Sasagawa, V. P. Pastukhov, K. Ishii, Y. Tatematsu, T. Saito, Md. K. Islam, Y. Nakashima, and T. Cho, International Conference on Open Magnetic Systems for Plasma Confinement, Novosibirsk, Russia, 5-9 July 2004 [Trans. Fusion Sci. Technol. 47, 276 (2005)].

${ }^{15}$ V. V. Arsenin and A. Yu. Kuyanov, Fiz. Plazmy 26, 793 (2000) [Plasma Phys. Rep. 26, 741 (2000)].

${ }^{16}$ V. V. Arsenin and A. Yu. Kuyanov, Fiz. Plazmy 27, 675 (2001) [Plasma Phys. Rep. 27, 635 (2001)].

${ }^{17}$ I. B. Bernstein, Advances in Plasma Physics, edited by A. Simon (Wiley,
New York, 1971), Vol. 4, p. 311.

${ }^{18}$ R. H. Cohen, Nucl. Fusion 19, 1579 (1979).

${ }^{19}$ D. D. Ryutov and G. V. Stupakov, Fiz. Plazmy 4, 501 (1977) [Sov. J. Plasma Phys. 4, 278 (1978)].

${ }^{20}$ D. D. Ryutov and G. V. Stupakov, Dokl. Akad. Nauk SSSR 240, 1086 (1978) [Sov. Phys. Dokl. 23, 412 (1978)].

${ }^{21}$ I. Katanuma, Y. Kiwamoto, S. Adachi, M. Inutake, K. Ishii, K. Yatsu, K. Sawada, and S. Miyoshi, Nucl. Fusion 27, 2041 (1987).

${ }^{22}$ I. Katanuma, Y. Kiwamoto, K. Ishii, K. Yatsu, and S. Miyoshi, Phys. Fluids B 1, 1459 (1989).

${ }^{23}$ J. R. Myra and P. J. Catto, Phys. Rev. Lett. 48, 620 (1982).

${ }^{24}$ T. Cho, M. Yoshida, J. Kohagura, M. Hirata, T. Numakura, H. Higaki, H. Hojo, M. Ichimura, K. Ishii, K. Md. Islam, A. Itakura, I. Katanuma, Y. Nakashima, T. Saito, Y. Tatematsu, M. Yoshikawa, Y. Kojima, S. Tokioka, N. Yokoyama, Y. Tomii, T. Imai, V. P. Pastukhov, S. Miyoshi, and GAMMA10 Group, Phys. Rev. Lett. 94, 085002-1 (2005).

${ }^{25}$ D. E. Baldwin and B. G. Logan, Phys. Rev. Lett. 43, 1318 (1979).

${ }^{26}$ T. C. Simonen, S. L. Allen, T. A. Casper, J. F. Clauser, C. A. Clower, F. H. Coensgen, D. L. Correll, W. F. Cummins, C. C. Damm, M. Flammer, J. H. Foote, R. K. Goodman, D. P. Grubb, E. B. Hooper, R. S. Hornady, A. L. Hunt, R. G. Kerr, A. W. Molvik, R. H. Munger, W. E. Nexsen, T. J. Orzechowkski, W. L. Pickles, P. Poulsen, M. E. Rensink, B. W. Stallard, W. C. Turner, W. L. Hsu, W. Bauer, T. L. Yu, and D. Zimmermann, Phys. Rev. Lett. 50, 1668 (1983).

${ }^{27}$ D. P. Grubb, S. L. Allen, T. A. Casper, J. F. Clauser, F. H. Coensgen, D. L. Correll, W. F. Cummins, C. C. Damm, J. H. Foote, R. K. Goodman, D. N. Hill, E. B. Hooper, Jr., R. S. Hornady, A. L. Hunt, R. G. Kerr, G. W. Leppelmeier, J. Marilleau, J. M. Moller, A. W. Molvik, W. E. Nexsen, W. L. Pickles, G. D. Porter, P. Poulsen, E. H. Silver, T. C. Simonen, B. W. Stallard, W. C. Turner, W. L. Hsu, T. L. Yu, J. D. Barter, T. Christensen, G. Dimonte, T. W. Romesser, R. F. Ellis, R. A. James, C. J. Lasnier, L. V. Berzins, M. R. Carter, C. A. Clower, B. H. Failor, S. Falabella, M. Flammer, and T. Nash, Phys. Rev. Lett. 53, 783 (1984).

${ }^{28}$ I. Katanuma, Y. Tatematsu, K. Ishii, T. Saito, and K. Yatsu, J. Plasma Fusion Res. 771085 (2001).

${ }^{29}$ I. Katanuma, Y. Tatematsu, K. Ishii, T. Saito, and K. Yatsu, Phys. Plasmas 9, 3449 (2002).

${ }^{30}$ I. Katanuma, Y. Tatematsu, K. Ishii, T. Saito, and K. Yatsu, Phys. Plasmas 10, 677 (2003). 